\title{
Thermo-Induced Aggregation and Crystallization of Block Copolypeptoids in Water
}

\author{
Christian Secker ${ }^{1}$, Antje Völkel ${ }^{1}$, Brigitte Tiersch ${ }^{2}$, Joachim Koetz $^{2}$, and Helmut Schlaad ${ }^{2 *}$ \\ ${ }^{1}$ Max Planck Institute of Colloids and Interfaces, Department of Colloid Chemistry, \\ Research Campus Golm, 14424 Potsdam, Germany. \\ ${ }^{2}$ University of Potsdam, Institute of Chemistry, Karl-Liebknecht-Straße 24-25, \\ 14476 Potsdam, Germany.
}

A)
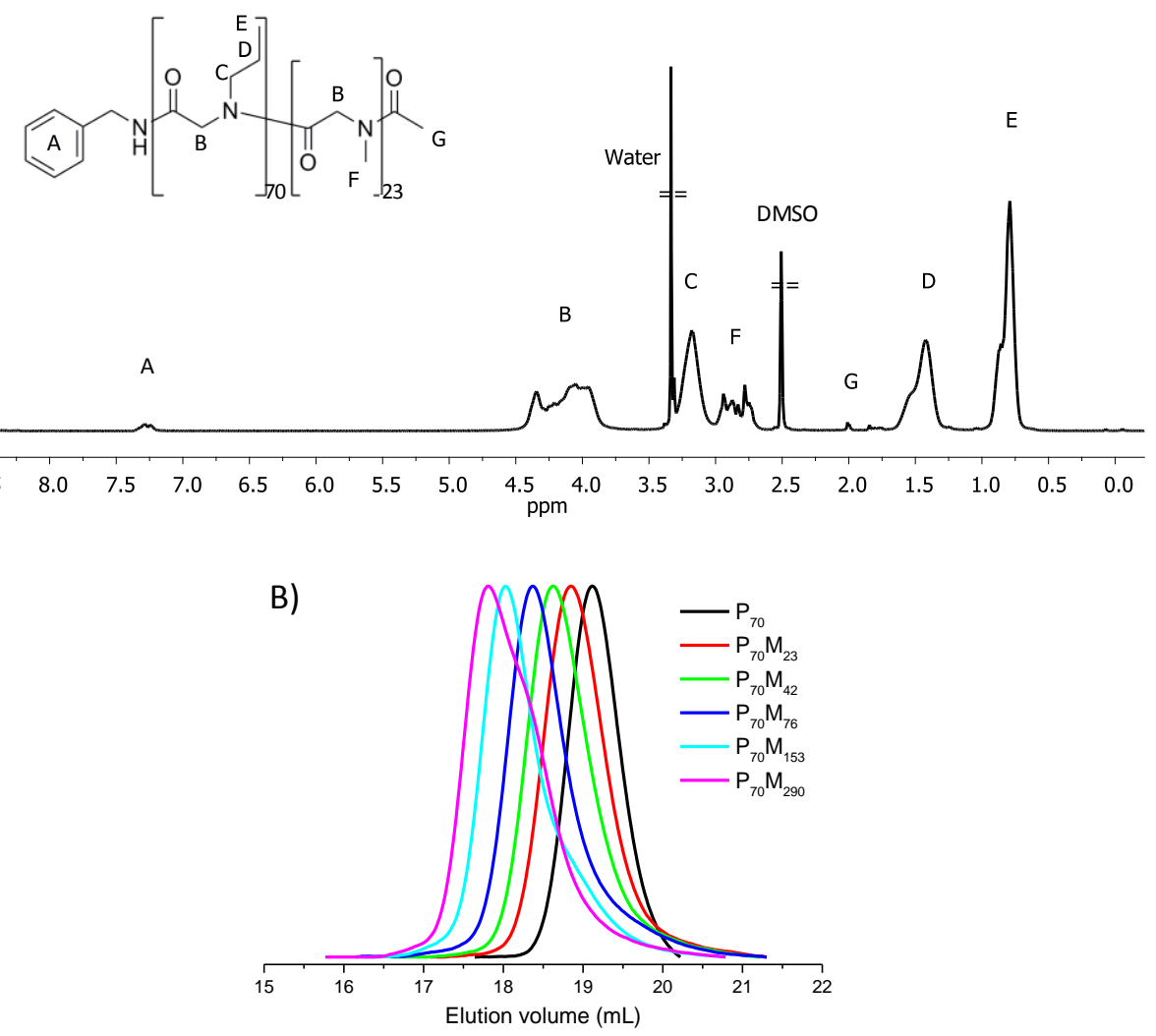

Figure S1. A) Exemplary ${ }^{1} \mathrm{H}-\mathrm{NMR}$ spectrum $(600 \mathrm{MHz})$ of $\mathrm{P}_{70} \mathrm{M}_{23}$ in $\mathrm{DMSO}-\mathrm{d}_{6}$ at room temperature. B) SEC RI traces (eluent: NMP) of the $\mathrm{P}_{70} \mathrm{M}_{\mathrm{m}}$ series. 


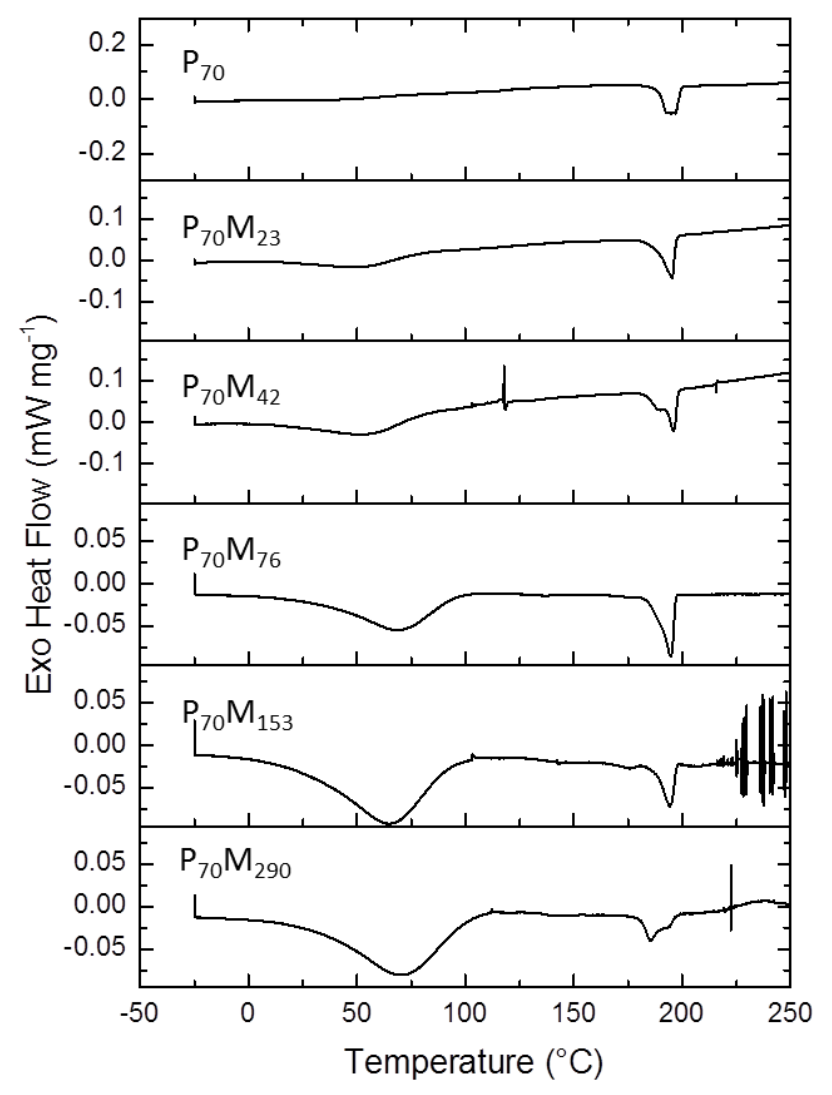

Figure S2. DSC first heating scans (heating rate: $1 \mathrm{~K} \mathrm{~min}^{-1}$ ) of the thermally annealed (in water) and freeze-dried block copolypeptoids $\mathrm{P}_{70} \mathrm{M}_{\mathrm{m}}$.

Table S1. Glass transition $\left(T_{\mathrm{g}}\right)$ and melting temperatures $\left(T_{\mathrm{m}}\right)$ of thermally annealed (in water) and freeze-dried block copolypeptoids $\mathrm{P}_{70} \mathrm{M}_{\mathrm{m}}$, as determined by DSC (Figure S2).

\begin{tabular}{lcl}
\hline Sample & $T_{\mathrm{g}}\left({ }^{\circ} \mathrm{C}\right)$ & \multicolumn{1}{c}{$T_{\mathrm{m}}\left({ }^{\circ} \mathrm{C}\right)$} \\
\hline $\mathrm{P}_{70}$ & 58 & $192,194,197$ \\
$\mathrm{P}_{70} \mathrm{M}_{23}$ & 65 & 193,195 \\
$\mathrm{P}_{70} \mathrm{M}_{42}$ & 68 & 187,194 \\
$\mathrm{P}_{70} \mathrm{M}_{76}$ & 84 & 188,194 \\
$\mathrm{P}_{70} \mathrm{M}_{153}$ & 82 & 194 \\
$\mathrm{P}_{70} \mathrm{M}_{290}$ & 89 & 185,194 \\
\hline
\end{tabular}


A)

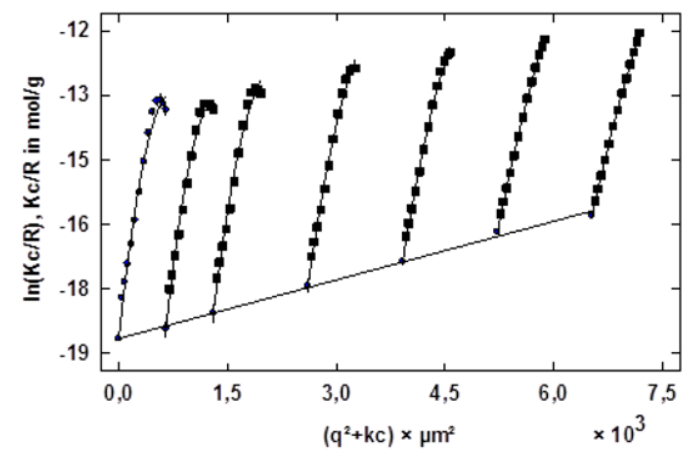

B)

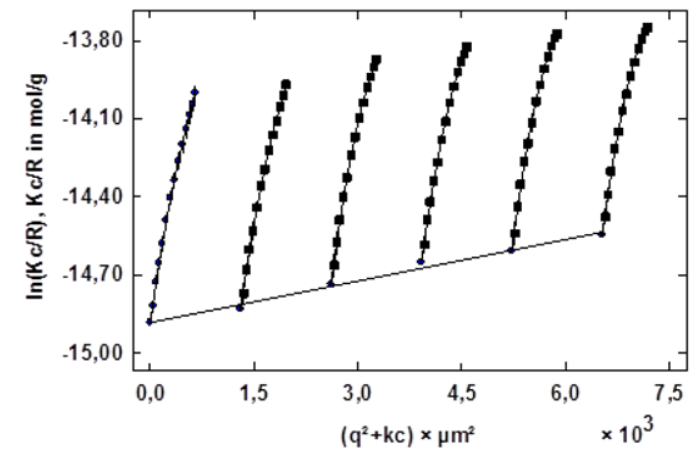

C)

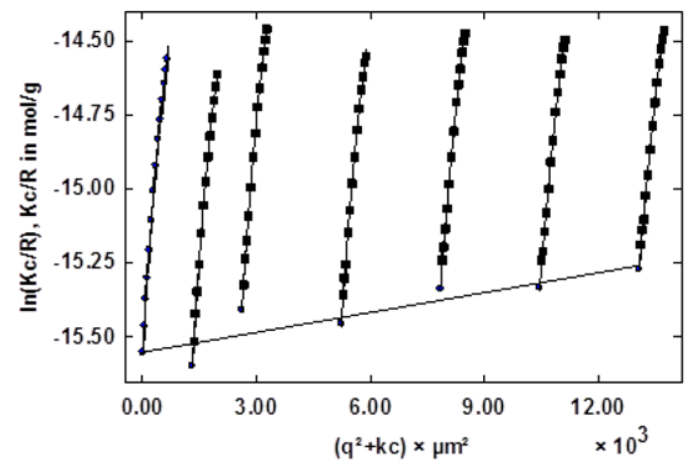

$\mathrm{M}_{\mathrm{w}}(0): 1.258 \mathrm{e}+08 \mathrm{~g} / \mathrm{mol}$

$\mathrm{M}_{\mathrm{w}}\left(\mathrm{q}^{2}\right): 1.258 \mathrm{e}+08 \mathrm{~g} / \mathrm{mol}$

A2: $1.179 \mathrm{e}-08 \mathrm{~mol} \mathrm{dm}{ }^{2} / \mathrm{g}^{2}$

$\mathrm{R}_{\mathrm{g}}: 2.167 \mathrm{e}+02 \mathrm{~nm}$

$\mathrm{M}_{\mathrm{w}}(0): 2.904 \mathrm{e}+06 \mathrm{~g} / \mathrm{mol}$

$\mathrm{M}_{\mathrm{w}}\left(\mathrm{q}^{2}\right): 2.904 \mathrm{e}+06 \mathrm{~g} / \mathrm{mol}$

A2: $5.972 \mathrm{e}-08 \mathrm{~mol} \mathrm{dm} \mathrm{dm}^{2} / \mathrm{g}^{2}$

$\mathrm{R}_{\mathrm{g}}: 7.452 \mathrm{e}+01 \mathrm{~nm}$

$M_{w}(0): 5.669 \mathrm{e}+06 \mathrm{~g} / \mathrm{mol}$

$M_{w}\left(q^{2}\right): 5.669 \mathrm{e}+06 \mathrm{~g} / \mathrm{mol}$

A2: $2.587 \mathrm{e}-08 \mathrm{~mol} \mathrm{dm} \mathrm{dm}^{2} / \mathrm{g}^{2}$

$\mathrm{R}_{\mathrm{g}}: 8.042 \mathrm{e}+01 \mathrm{~nm}$

Figure S3. SLS Guinier plots for 0.01-0.1 wt \% aqueous dispersions of thermally annealed block copolypeptoids A) $\mathrm{P}_{70} \mathrm{M}_{23}$, B) $\mathrm{P}_{70} \mathrm{M}_{42}$, and C) $\mathrm{P}_{70} \mathrm{M}_{76}$. 